\title{
Erratum to: Hypoxia Augments Increased HIF-1a and Reduced Survival Protein p-Akt in Gelsolin (GSN)-Dependent Cardiomyoblast Cell Apoptosis
}

\author{
Yu-Lan Yeh ${ }^{1,2}$ - Wei-Syun Hu ${ }^{3,4}$ - Wei-Jen Ting ${ }^{5}$ Chia-Yao Shen ${ }^{6}$ Hsi-Hsien Hsu ${ }^{7}$. \\ Li-Chin Chung ${ }^{8}$ Chuan-Chou Tu' ${ }^{9}$ Sheng-Huang Chang ${ }^{10}$ - Cecilia-Hsuan Day ${ }^{6}$. \\ Yuhsin Tsai ${ }^{11} \cdot$ Chih-Yang Huang ${ }^{5,11,12}$
}

Published online: 3 October 2017

(C) Springer Science+Business Media, LLC 2017

Erratum to: Cell Biochem Biophys (2016) 74:221-228 DOI 10.1007/s12013-016-0729-6

The original version of this article unfortunately contained an error. One of the authors, Dr. Wei-Syun Hu, was inadvertently omitted from the author group. The missing author's name and affiliation are now included in this erratum. The authors regret the oversight. Also, Yu-Lan Yeh and Wei-Syun Hu contributed equally to this work.
The online version of the original article can be found at doi:10.1007/ s12013-016-0729-6.

Yu-Lan Yeh and Wei-Syun Hu contributed equally to this work.

Chih-Yang Huang

cyhuang@mail.cmu.edu.tw

1 Department of Pathology, Changhua Christian Hospital, Changhua, Taiwan

2 Department of Medical Technology, Jen-Teh Junior College of Medicine, Nursing and Management, Miaoli, Taiwan

3 PhD Program for Aging, China Medical University, Taichung 40402, Taiwan

4 Division of Cardiovascular Medicine, Department of Medicine, China Medical University Hospital, Taichung 40447, Taiwan

5 Graduate Institute of Basic Medical Science, School of Chinese Medicine, China Medical University and Hospital, 91 Hsueh-Shih Road 404, Taichung, Taiwan, ROC

6 Department of Nursing, MeiHo University, Pingtung, Taiwan
Division of Colorectal Surgery, Mackay Memorial Hospital, Taipei, Taiwan

8 Department of Hospital and Health Care Administration, Chia Nan University of Pharmacy and Science, Tainan County, Taiwan

9 Division of Chest Medicine, Department of Internal Medicine, Armed Force Taichung General Hospital, Taichung, Taiwan

10 Department of Health, Tsao-Tun Psychiatric Center, Executive Yuan, Nantou 54249, Taiwan

11 School of Chinese Medicine, China Medical University, Taichung, Taiwan

12 Department of Health and Nutrition Biotechnology, Asia University, Taichung, Taiwan 